\title{
Effect of Hospital Characteristics on the Quality of Laparoscopic Gastrectomy in Japan
}

\author{
Kazuaki Kuwabara, ${ }^{\mathrm{a}, \mathrm{g}}$, Shinya Matsuda ${ }^{\mathrm{b}}$, Kiyohide Fushimi ${ }^{\mathrm{c}}$, Koichi B Ishikawa ${ }^{\mathrm{d}}$, \\ Hiromasa Horiguchi ${ }^{e}$, Kenji Fujimorif ${ }^{\mathrm{f}}$
}

\begin{abstract}
Background: Laparoscopic gastrectomy (LG) is becoming more widely indicated, although its application has not been investigated sufficiently in community-based gastrointestinal research because the small number of gastric cancers in western countries might have limited its use. However, concerns have been raised regarding variations in the quality of care with LG. To contribute to improving the efficient utilization of costly surgical innovations, we determined the impact of hospital characteristics on LG care.
\end{abstract}

Methods: Among 3,914 LG patients in 58 academic and 200 community hospitals between 2006 and 2008, we examined patient demographics, comorbidity, complications, partial or total gastrectomy, care process, hospital patient volume, hospital ownership and teaching status, and fiscal year. Hospital LG volume was divided into three quintile categories (lower volume, LV; intermediate volume, IV; or higher volume, HV) that consisted of an approximately equal number of patients. Dependent variables were operating time (OT), length of stay (LOS) and total charge (TC). The impact of hospital characteristics on these variables was assessed using multivariate analysis.

Manuscript accepted for publication April 20, 2010

\footnotetext{
${ }^{a}$ Kyushu University Graduate School of Medical Sciences, Department of Health Care Administration and Management, 3-1-1 Maidashi, Hi gashi-ku, Fukuoka, Japan 812-8582

bUniversity of Occupational and Environmental Health, 1-1 Iseigaoka

Yahatanishi-ku Kitakyushu, Fukuoka 807-8555, Japan

'Tokyo Medical and Dental University, 1-5-45 Yushima Bunkyo-ku, To kyo 113-8510, Japan

${ }^{d}$ National Cancer Center, 5-1-1 Tsukiji Chuo-ku, Tokyo 104-0045, Japan eUniversity of Tokyo Graduate School of Medicine, 7-3-1 Hongo Bun kyo-ku, Tokyo 113-0033, Japan

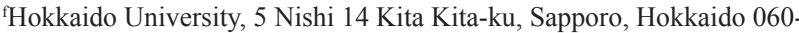
8648, Japan

${ }^{g}$ Corresponding author: Kazuaki Kuwabara, Kyushu University Graduate School of Medical Sciences, Department of Health Care Administra tion and Management, 3-1-1 Maidashi, Higashi-ku, Fukuoka 812-8582, Japan.Email: kazu228@basil.ocn.ne.jp
}

doi:10.4021/gr2010.04.189w
Results: Twenty-seven academic hospitals out of 193 LV hospitals treated $271(21 \%)$ LG patients, 20 of 44 IV hospitals treated 596 (47\%), and 11 of $21 \mathrm{HV}$ hospitals treated 748 (55\%). Although mortality or complications did not vary significantly between LV, IV and HV hospitals, the latter were associated with longer OT or LOS and more TC. More blood transfusions were required in HV hospitals once indicated. Hospital ownership or teaching status did not explain the variation in complications. Teaching and national hospitals consumed more resources, and municipal and private hospitals reduced OT more than national hospitals.

Conclusions: A volume-quality relationship was recognized. As intraoperative transfusion prolongs OT and results in more complications, clinical societies or policy makers should introduce this new technique concurrently with quality improvement initiatives that aim to reduce unnecessary OT at targeted institutions. Hospitals varied in terms of LOS and TC, therefore, policy makers should also monitor resource utilization to enhance the efficiency of LG care under restrictive fiscal policies.

Keywords: Hospital volume; Laparoscopic gastrectomy; Length of stay; Operating time; Quality of care

\section{Introduction}

Gradual confirmation of the safety and superiority of laparoscopic gastrectomy (LG) over conventional open gastrectomy (OG) has expanded the indications for LG in patients with advanced-stage gastric cancer, or those admitted to community hospitals [1-3]. As the estimated annual number of LGs in Japan has increased from around 3,600 in 2006 to 9,600 in 2007, these cases have provided cumulative clinical and claims data, which have enabled quantitative analysis of the quality of LG, and identification of hospital characteristics associated with quality of LG care, through an administrative database $[4,5]$.

Several studies have evaluated factors that affect the utilization of technical innovations, including laparoscopic surgery or diagnostic imaging [6-11]. Hospital factors such as volume, geographic region, ownership, teaching status, or 
socioeconomic status have been observed to explain variations in the quality of surgical innovation. Population studies using the administrative database have contributed toward those observations.

However, application of LG has not been investigated sufficiently in community-based studies because the smaller number of gastric cancers in western countries might have limited identification of variation in LG use [7]. As LG use has increased in Japan, community-based studies of variation in quality of care should be carried out by focusing on hospital characteristics. The findings derived from such studies could contribute to improving the utilization of costly surgical innovations such as LG, and policy-makers could play a definitive role in quality improvement initiatives through implementation of restrictive fiscal policies.

In this study, we determined the impact of hospital characteristics on complications, resource use and care of LG patients in Japan.

\section{Materials and Methods}

We used a Japanese administrative database that was incorporated into the Ministry of Health, Labor and Welfare (MHLW) and our own research project that was concerned with the development of a Japanese case-mix classification system. Up to 82 academic and 1,346 community hospitals were enrolled in this project in 2008. Anonymous health insurance claims data with detailed clinical information have been collected annually for a study period of 6 months from July 1, 2006 for this database, and were provided to our research team. This database contained details of the date and quantity of care provided during hospitalization, therefore, it was used to assess hospital performance and payments by MHLW. Among a total of 8,010,361 patients from 1,006 hospitals that voluntarily participated in our research project, we analyzed gastrectomy patients treated consecutively in 258 hospitals from fiscal year (FY) 2006 to 2008. Our project was approved by the ethical committee of the University of Occupational and Environmental Health, Fukuoka, Japan.

\section{Variable definitions}

Study variables included age, sex, discharge outcome, pathology of gastric disease (benign or malignant), comorbidity, physical status classification by the American Society of Anesthesiologists (ASA), gastrectomy type (partial or total gastrectomy), procedure-related complications, chemotherapy, care process (fasting day, day of postoperative epidural anesthesia, blood transfusion, use or number of circular or linear stapling devices fired), hospital LG volume, hospital ownership and teaching status, and FY. We also calculated length of stay (LOS), total charges (TCs; US\$1= $¥ 100$ ) and operating time (OT). TCs included fees for physician consul- tation and administration, and costs of instruments, laboratory tests and imaging. TC, which is billed during admission, is considered to be a good estimate of healthcare costs [12]. OT included the induction of general anesthesia and preparation for video-monitoring and surgical procedures, in addition to skin to skin time.

Patients were stratified into two age groups: $<65$ years, and $\geq 65$ years. Diagnosis was coded by the International Classification of Disease 10th version (ICD code). Malignant diseases were referred to as $\mathrm{C} 16$ and $\mathrm{C} 170$, and benign diseases as D131-2, D371, K25-6, and K31. The database recorded a maximum of either four comorbidities or four complications per patient.

To assess the severity of preexisting comorbid conditions, we used the Charlson comorbidity index (CCI) [13]. Patients were divided into four groups: $\mathrm{CCI}=0,1,2$ or $\geq 3$. ASA score was summarized into three groups: 1,2 or $\geq 3$. Procedure-related complications included wound complications, hematoma or others (T81 - T87), bowel obstruction (K565-7 and K913), and acute pancreatitis (K85) [14]. Use of chemotherapy or preoperative blood transfusion was a proxy for disease severity, advanced gastric cancer or preexisting anemia. Gastrectomy type was recorded by the Japanese original claim code for which gastrectomy was categorized as partial or total or as OG or LG.

Hospital LG volume was divided into three quintile categories (lower volume, LV; intermediate volume, IV; or higher volume, HV) that consisted of an approximately equal number of patients. Hospital ownership was classified into four groups (national, municipal, non-profit private, and private for profit). Non-profit private hospitals included Red Cross Hospitals and charity hospitals. The remaining private hospitals were designated as private for profit (Japanese legislation prohibits them to have stock holders). Teaching hospitals were responsible for the clinical research as well as education of medical students and postgraduate trainees.

\section{Statistical analysis}

Frequencies and proportions for categorical data were compared by Fisher's exact test. Continuous variables were compared between LV, IV and HV hospitals using analysis of variance. Logistic regression was used to evaluate the association of hospital characteristics and volume with complications. Multiple linear regression analysis was employed to assess the effect of hospital variables on OT, LOS and TC. Statistical analysis was performed using SPSS version 16.0, with a level of significance set at $\mathrm{p}<0.05$.

\section{Results}

The 58 academic hospitals (27 LV, 20 IV and $11 \mathrm{HV}$ ) and 200 community hospitals (166 LV, 24 IV and 10 HV) in- 


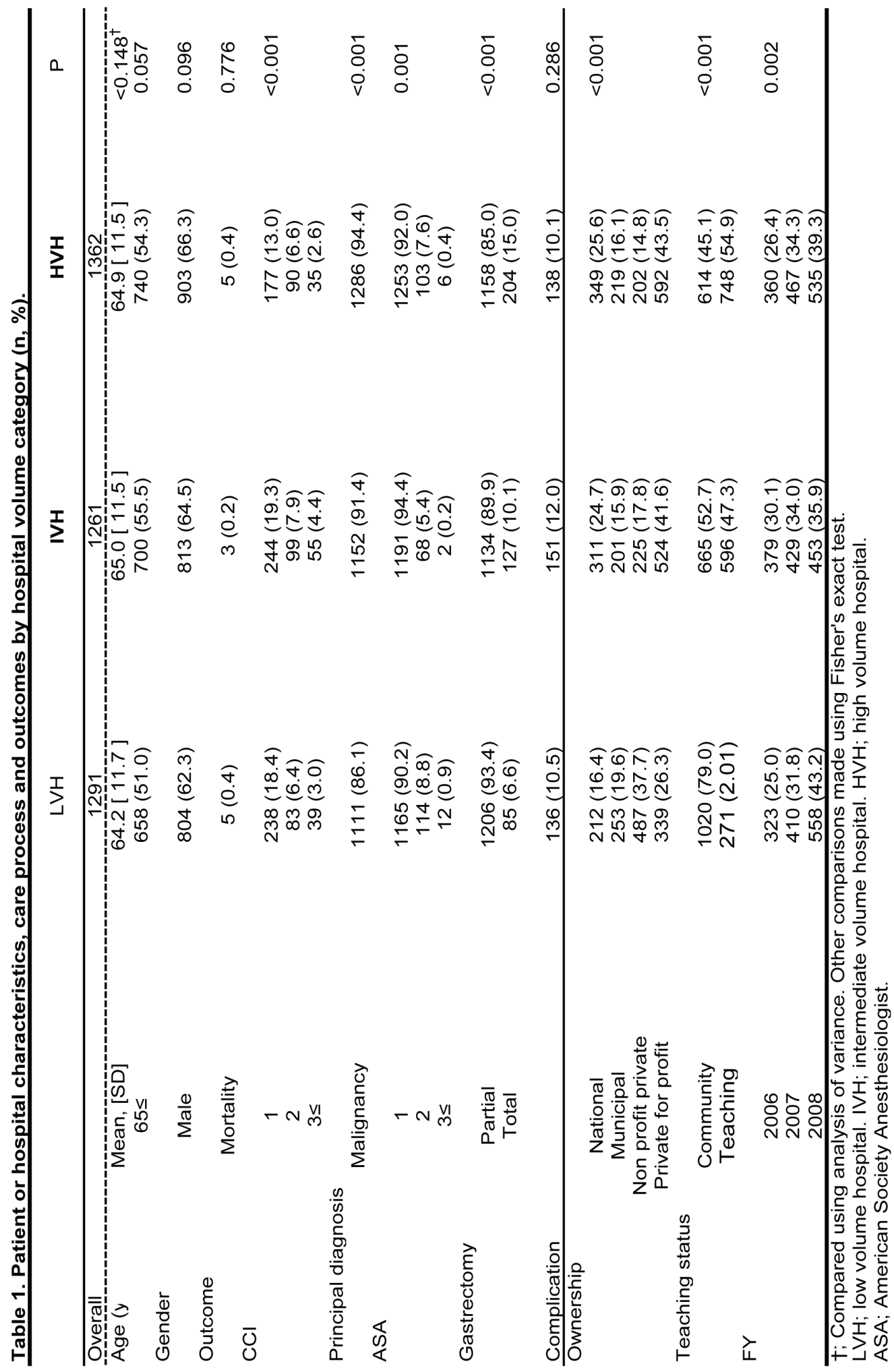




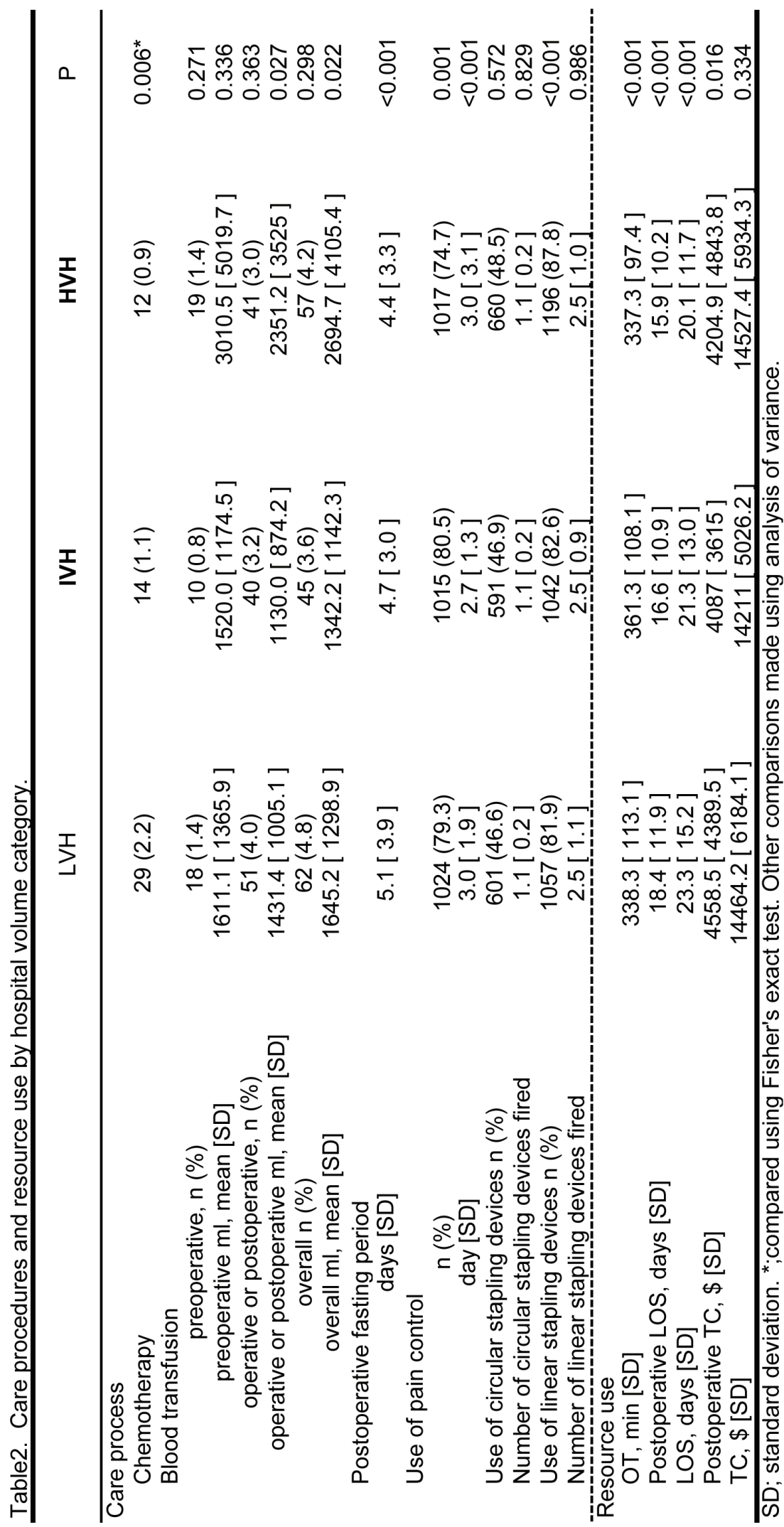


cluded a total of 3,914 LG patients. LV hospitals performed a mean 4.5 LG procedures annually (range 0.7 - 12.7); IV hospitals performed a mean 19.1 (13.3 - 25.3); and HV hospitals performed a mean $43.2(26-106.7)$. As for ownership, 40 national hospitals (22 LV, $11 \mathrm{IV}$ and $7 \mathrm{HV}$ ) provided care for a mean 14.5 LG patients annually (range 0.7 - 52.0); $52 \mathrm{mu}$ nicipal hospitals (41 LV, 7 IV and $4 \mathrm{HV}$ ) cared for 8.6 (0.7 43.3); 79 non-profit private hospitals (67 LV, 9 IV and $3 \mathrm{HV}$ ) cared for 7.7 (0.7 - 48.7); and 87 private for profit hospitals (63 LV, $17 \mathrm{IV}$ and $7 \mathrm{HV}$ ) cared for $11.1(0.7$ - 106.7) patients.

There was no significant difference in age, sex, mortality and complications between hospital volume categories. Patients with less comorbidity or more malignant disease, and more indications for total gastrectomy were observed in HV hospitals. Private hospitals used LG more than national and municipal hospitals. As FY progressed, more LG was indicated in every volume category (Table 1).

The average number of operative/postoperative or overall blood transfusions was greater in HV hospitals, although the proportion of transfusions did not differ significantly among the hospital volume categories. The number of patients undergoing chemotherapy and postoperative pain control was lower in HV hospitals, whereas the proportion of linear stapling devices fired was higher. Postoperative fasting days and OT were shorter in HV hospitals. LOS and TC were significantly greater in LV hospitals, except for TC during hospitalization $(\mathrm{p}=0.334)$ (Table 2).

Longer OT and lower hospital volume were found to be significant predictors of more complications, for which hospital ownership and teaching status were not significant determinants. FY 2008 was associated significantly with more complications (Table 3).

Lower patient volume and national hospitals were associated significantly with longer OT. Higher patient volume, municipal or private for profit hospitals were significant determinants of shorter LOS and lower TCs, whereas academic hospitals had longer LOS and higher TCs. FY was not a determinant of variation in OT and TC (Table 4).

\section{Discussion}

This study revealed that higher-volume hospitals achieved more efficient LG care, as well as fewer complications. Significant variation in OT and resource use was observed in relation to hospital ownership and teaching status, however, rate of complications was not so affected. FY was another independent predictor of complications.

There have been various kinds of innovations in laparoscopy-assisted surgery; newer techniques like laparoscopic nephrectomy, adrenalectomy or LG in the present study on the one hand, and more established procedures like laparoscopic cholecystectomy on the other hand, which has been employed for more complicated cases as a result of its proven safety record $[7,10,11,15]$. Once the feasibility, safety and novelty of these procedures had been confirmed, clinical societies attempted to make them widely available by promoting surgical skills or encouraging their application through health policy initiatives.

To the best of our knowledge, previous studies have not been conducted in western countries because of the relative infrequency of gastric cancer compared with colorectal malignancies [7]. Reid-Lombard et al have discussed the volume-quality relationship in gastrectomy and have supported evidence-based hospital transfer, for which high gastrectomy volume, irrespective of LG or OG, was designated as $>9$ to $>50$ cases per year [7]. This corresponded to LV to HV hospitals in our study. Even though we acknowledge the difference in the definition of hospital categories, volume-quality relationships in terms of complications and resource use should be addressed as a worldwide concern. HV hospitals did demonstrate superiority in terms of complication rates and efficient care of LG patients over LV and IV hospitals. However, teaching hospitals were associated with greater resource use, but not with complications or longer OT. This might have been because teaching hospitals attempted to provide education in the skills needed for LG by offsetting this against the cost of providing efficient LG care [16]. HV hospitals required a greater number of operative/postoperative blood transfusions, although the frequency of transfusion was lower than that in LV or IV hospitals. The need for more blood transfusions might have arisen from intractable bleeding from splenic tears, which resulted in longer operations and a greater frequency of complications, because operative transfusion proxy of bleeding proved to prolong OT (Table 4). This is one reason why quality improvement initiatives or perioperative multidisciplinary care should be progressed. The appropriate application of stapling devices to prevent accidental bleeding, or a curriculum to educate surgeons and their staff teams about more practical handling devices should be considered $[17,18]$.

When studying the dissemination of technological innovations like magnetic resonance imaging (MRI) and laparoscopic surgery, or variations in their indications, hospital characteristics and socioeconomic status have always been included in the analytical model [6-11]. Among these, hospital or surgeon volume, and hospital ownership and teaching status have been well documented $[8,19]$. In our study, private hospitals were not inclined to make the resources available, although they were able to reduce OT and not increase the rate of complications. Hahm et al have reported that the ownership relationship is significant for MRI [8]. They wish to enhance the reputation of LG by establishing its safety and efficacy, and developing more costly infrastructure, including video-monitoring, skilled staff and disposable devices, this is another justification for implementation of an efficient fiscal policy. Municipal hospitals consumed fewer resources in the present study; therefore, accountability for greater re- 
Table 3. Factors associated with complication.

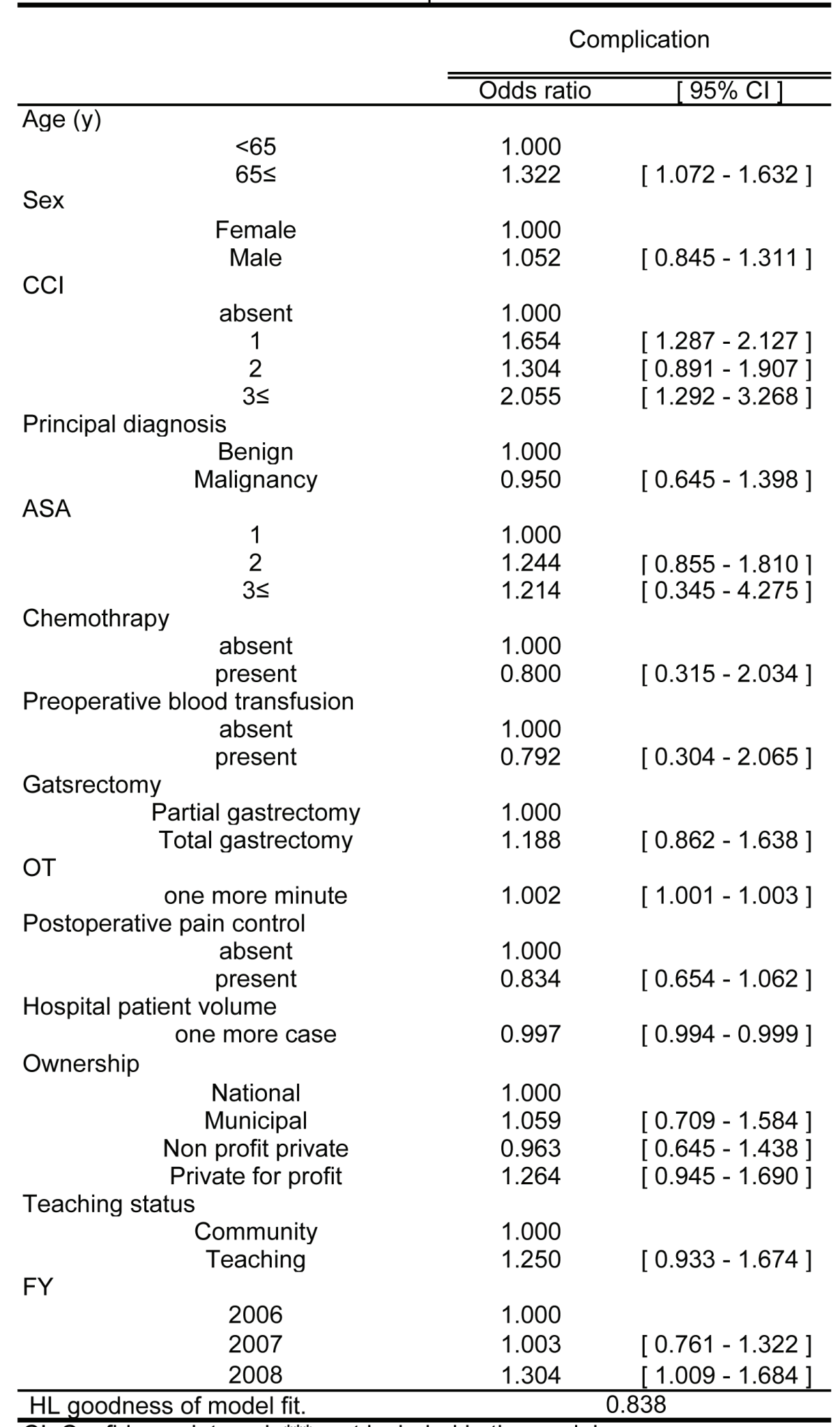

$\mathrm{Cl}$; Confidence interval. ${ }^{* * *}$; not included in the model.

ASA; American Society Anesthesiologist. HL; Hosmer Lemeshow. 


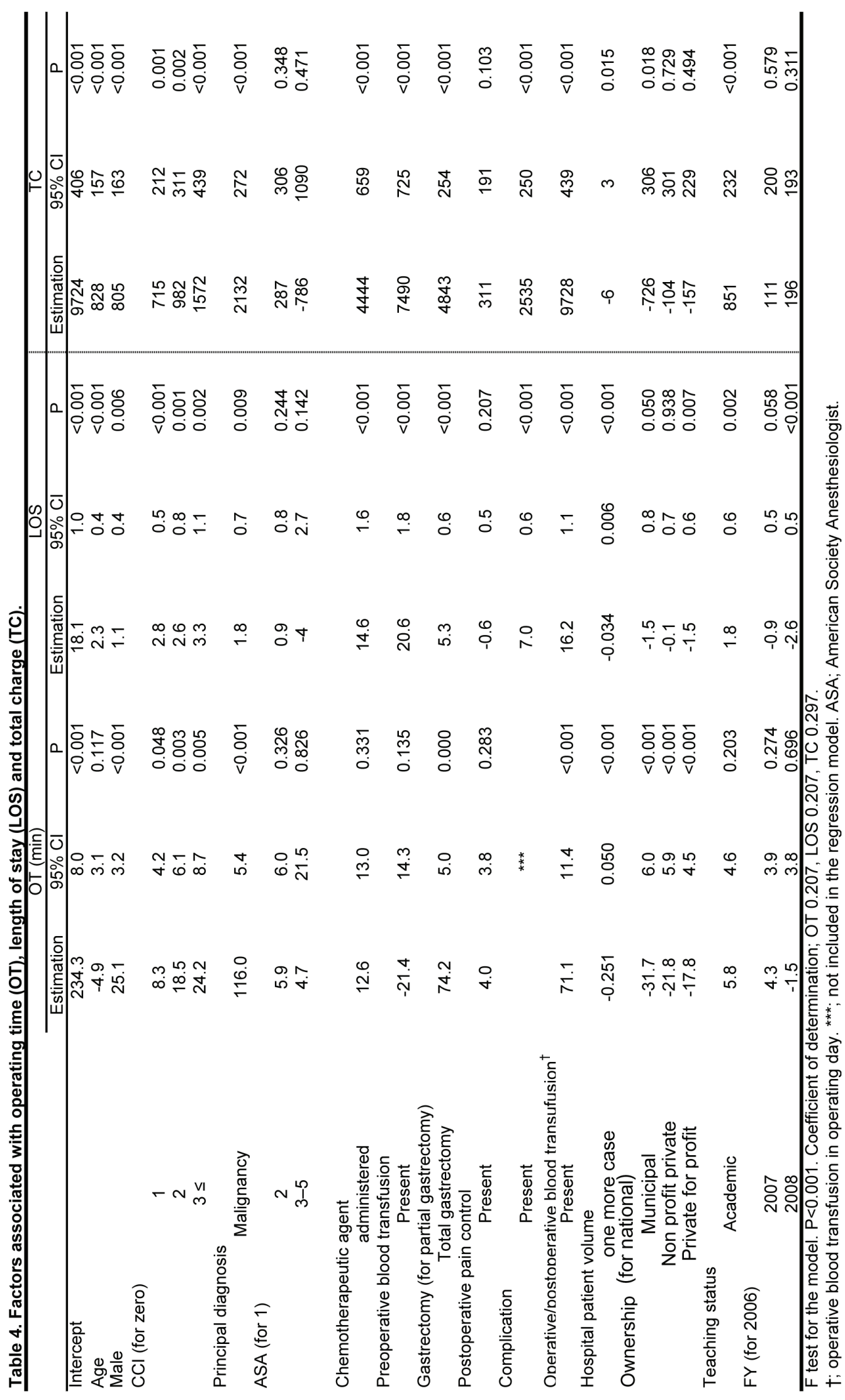


source spending might be requested in private or academic hospitals [7-11].

There are several limitations to the present study. First, the study period was limited to only 6 months, which might diminish the generalization of our results. Our study sample size, which was representative of one third to half of LG procedures performed in Japan, assured the validity of our study, if we include all hospitals that participated in our project. The exhaustive MHLW database, for which the study period is planned to be extended by up to 12 months in 2010 , should also overcome this limitation. Second, LOS in Japan remains generally 2 - 3 times longer than that in Western countries [20]. Japanese hospitals generally provide wound management and nursing home services in addition to acute medical care. Accordingly, our results might actually reflect the real costs incurred during the entire care process [21]. Third, some clinical information such as body mass index (BMI), cancer staging, and extent of lymphadenectomy, was not recorded. Organization for Economic Co-operation and Development (OECD) health data for 2002 have demonstrated that mean BMI was $\leq 23.4$ in East Asian people [20], which should not have affected the results of the present study. Administration of chemotherapy was defined as a marker of advanced cancer stage. More complications were found in FY 2008, and extended lymphadenectomy, which is often considered to be associated with postoperative complications, including intraoperative bleeding sufficiently severe to require intraoperative/postoperative blood transfusion, might be more undertaken during LG [22]. Cancer registry, including the extent of lymphadenectomy, accompanied by this administrative database could measure the effect of this clinical information on resource use and the care process, and enhance the findings of the volume-quality relationship.

In conclusion, our analysis demonstrated that hospital patient volume was associated with fewer complications, shorter LOS and lower TCs. Teaching hospitals were associated with longer OT and LOS and higher TCs. Private hospitals did not reduce the resource use in spite of the ability to perform shorter operations. The number of transfusions was lower in HV hospitals, but they required more transfusion once indicated. Intraoperative transfusion prolonged OT, which possibly resulted in more complications. Therefore, clinical societies or policy makers should endeavor to introduce this new technique concurrently with quality improvement initiatives aimed at the targeted institutions, to enhance the efficiency of LG care, and to encourage the development of surgical skills to prevent unexpected bleeding.

\section{Acknowledgments}

This study was funded in part by Grants-in-Aid for Research on Policy Planning and Evaluation from the Ministry of Health, Labour and Welfare, Japan (H19 Seisaku-sitei 001).

\section{Financial Disclosure}

All authors declared no conflict of interest for this submission.

\section{References}

1. Kitano S, Iso Y, Moriyama M, Sugimachi K. Laparoscopy-assisted Billroth I gastrectomy. Surg Laparosc Endosc 1994;4(2):146-148.

2. Kitano S, Shiraishi N, Uyama I, Sugihara K, Tanigawa N. A multicenter study on oncologic outcome of laparoscopic gastrectomy for early cancer in Japan. Ann Surg 2007;245(1):68-72.

3. Hwang SI, Kim HO, Yoo CH, Shin JH, Son BH. Laparoscopic-assisted distal gastrectomy versus open distal gastrectomy for advanced gastric cancer. Surg Endosc 2009;23(6):1252-1258.

4. Ministry of Health, Welfare and Labor. Survey of National Medical Care Insurance Services 2006 (in Japanese). Tokyo, Japan: Health and Welfare Statistics Association. Available at http://www.dbtk.mhlw.go.jp/ IPPAN/ippan/scm_k_Ichiran (Date accessed February 16).

5. Ministry of Health, Welfare and Labour. Survey of National Medical Care Insurance Services 2007 (in Japanese). Tokyo, Japan: Health and Welfare Statistics Association. Available at http://www.dbtk.mhlw.go.jp/ IPPAN/ippan/scm_k_Ichiran (accessed February 16).

6. Weber WP, Guller U, Jain NB, Pietrobon R, Oertli D. Impact of surgeon and hospital caseload on the likelihood of performing laparoscopic vs open sigmoid resection for diverticular disease: a study based on 55,949 patients. Arch Surg 2007;142(3):253-259; discussion 259.

7. Reid-Lombardo KM, Gay G, Patel-Parekh L, Ajani JA, Donohue JH. Treatment of gastric adenocarcinoma may differ among hospital types in the United States, a report from theNational Cancer Data Base. J Gastrointest Surg 2007;11(4):410-419; discussion 419-420.

8. Hahm MI, Park EC, Lee SH, Nam CM, Kang HY, Lee HY, Cho WH. Pattern and factors leading to the diffusion of magnetic resonance imaging in Korean hospitals. Int J Technol Assess Health Care 2007;23(2):292-298.

9. Miller DC, Saigal CS, Banerjee M, Hanley J, Litwin MS. Diffusion of surgical innovation among patients with kidney cancer. Cancer 2008;112(8):1708-1717.

10. Miller DC, Daignault S, Wolf JS, Jr., Hafez KS, Kieran K, Dunn RL, Hollenbeck BK. Hospital characteristics and use of innovative surgical therapies among patients with kidney cancer. Med Care 2008;46(4):372-379.

11. Henneman D, Chang Y, Hodin RA, Berger DL. Effect of laparoscopy on the indications for adrenalectomy. Arch 
Surg 2009;144(3):255-259; discussion 259.

12. Hayashida K, Imanaka Y, Otsubo T, Kuwabara K, Ishikawa KB, Fushimi K, Hashimoto H, et al. Development and analysis of a nationwide cost database of acute-care hospitals in Japan. J Eval Clin Pract 2009;15(4):626633.

13. Sundararajan V, Henderson T, Perry C, Muggivan A, Quan H, Ghali WA. New ICD-10 version of the Charlson comorbidity index predicted in-hospital mortality. $\mathrm{J}$ Clin Epidemiol 2004;57(12):1288-1294.

14. Zhan C, Miller MR. Administrative data based patient safety research: a critical review. Qual Saf Health Care 2003;12 Suppl 2(ii58-63.

15. Johansson M, Thune A, Nelvin L, Stiernstam M, Westman B, Lundell L. Randomized clinical trial of open versus laparoscopic cholecystectomy in the treatment of acute cholecystitis. Br J Surg 2005;92(1):44-49.

16. Nguyen N, Yegiyants S, Kaloostian C, Abbas MA, Difronzo LA. The Surgical Care Improvement project (SCIP) initiative to reduce infection in elective colorectal surgery: which performance measures affect outcome? Am Surg 2008;74(10):1012-1016.

17. Dowdall JF, McAnena OJ. Linear stapling of the short gastric vessels reduces blood loss and splenectomy at oesophageal and gastric surgery. Surg Endosc 2006;20(5):770-772.

18. Scott DJ, Dunnington GL. The new ACS/APDS Skills Curriculum: moving the learning curve out of the operating room. J Gastrointest Surg 2008;12(2):213-221.

19. Lin HC, Xirasagar S, Lee HC, Chai CY. Hospital volume and inpatient mortality after cancer-related gastrointestinal resections: the experience of an Asian country. Ann Surg Oncol 2006;13(9):1182-1188.

20. OECD Health Data 2008 - Frequently Requested Data. Available from http://www.oecd.org/document/16/0,3 343,en_2649_34631_2085200_1_1_1_1,00.html (accessed 4 November 2008).

21. Ishizaki T, Imanaka Y, Hirose M, Kuwabara K, Ogawa T, Harada Y. A first look at variations in use of breast conserving surgery at five teaching hospitals in Japan. Int J Qual Health Care 2002;14(5):411-418.

22. Bonenkamp JJ, Songun I, Hermans J, Sasako M, Welvaart K, Plukker JT, van Elk P, et al. Randomised comparison of morbidity after D1 and D2 dissection for gastric cancer in 996 Dutch patients. Lancet 1995;345(8952):745748. 